\title{
The Use of Zein and Shuanghuangbu for Periodontal Tissue Engineering
}

\author{
Yan-zhi Xu*, Jing-jing Wu, Yan-ping Chen, Jian Liu, Na Li, Feng-ying Yang \\ Department of Stomatology, the Fourth Hospital of Hebei Medical University, Shijiazhuang, China
}

\begin{abstract}
Aim Tissue engineering is a promising area with a broad range of applications in the fields of regenerative medicine and human health. The emergence of periodontal tissue engineering for clinical treatment of periodontal disease has opened a new therapeutic avenue. The choice of scaffold is crucial. This study was conducted to prepare zein scaffold and explore the suitability of zein and Shuanghuangbu for periodontal tissue engineering.
\end{abstract}

Methodology A zein scaffold was made using the solvent casting/particulate leaching method with sodium chloride $(\mathrm{NaCl})$ particles as the porogen. The physical properties of the zein scaffold were evaluated by observing its shape and determining its pore structure and porosity. Cytotoxicity testing of the scaffold was carried out via in vitro cell culture experiments, including a liquid extraction experiment and the direct contact assay. Also, the Chinese medicine Shuanghuangbu, as a growth factor, was diluted by scaffold extract into different concentrations. This Shuanghuangbu-scaffold extract was then added to periodontal ligament cells (PDLCs) in order to determine its effect on cell proliferation.

Results The zein scaffold displayed a sponge-like structure with a high porosity and sufficient thickness. The porosity and pore size of the zein scaffold can be controlled by changing the porogen particles dosage and size. The porosity was up to $64.1 \%-78.0 \%$. The pores were well-distributed, interconnected, and porous. The toxicity of the zein scaffold was graded as $0-1$. Furthermore, PDLCs displayed full stretching and vigorous growth under scanning electronic microscope (SEM). Shuanghuangbu-scaffold extract could reinforce proliferation activity of PDLCs compared to the control group, especially at $100 \mu \mathrm{g} \cdot \mathrm{mL}^{-1}(P<0.01)$.

Conclusion A zein scaffold with high porosity, open pore wall structure, and good biocompatibility is conducive to the growth of PDLCs. Zein could be used as scaffold to repair periodontal tissue defects. Also, Shuanghuangbuscaffold extract can enhance the proliferation activity of PDLCs. Altogether, these findings provide the basis for in vivo testing on animals.

Keywords zein, periodontal tissue engineering, scaffold, periodontal ligament cells, Shuanghuangbu

Received Jun. 21, 2010; Revision accepted Aug. 20, 2010

\section{Introduction}

Periodontal disease is the primary cause of periodontal tissue defects, often leading to tooth loss in adults. The functional decline of teeth seriously affects health and quality of life (You et al., 2009). For a long time, researchers have been trying to find innovative methods to effectively increase new attachment formation on teeth or reconstruct periodontal supporting tissues in order to treat periodontal disease. Although there has been some progresses in the treatment of periodontal defects, the available approaches still need improvement for reconstructing periodontal tissues. In recent years, the emergence of periodontal tissue engineering technology has opened up a new approach to the clinical treatment of periodontal diseases (Chen and Yan, 2010). An important prerequisite for tissue engineering is to find the appropriate scaffold material. Collagen (Dawson et al., 2008), PLLA (Montjovent et al., 2008) and hydroxyapatite (Thein Han et al., 2009) have been used as scaffolds in tissue engineering. However, there are still many disadvantages using these materials. Therefore, obtaining the desirable scaffold material remains a high priority. 
Zein is a mixture of alcohol soluble proteins that constitute $50 \%-60 \%$ of total endosperm protein. Because zein generally lacks lysine, tryptophan, and other human essential amino acids, its edible and nutritional value is relatively low. However, zein has a unique solubility, heat resistance, filmformation, antimicrobial properties, and anti-oxidative properties. It can be used for preventing moisture, isolating oxygen, as an anti-UV agent, an anti-static agent, and so on. Due to these characteristics, it has been the focus of exploration and application in many research areas. Studies have shown that zein is an important resource for the production of edible biodegradable food packaging materials ( $\mathrm{Ku}$ and Song, 2007). The good antimicrobial and film-forming properties of zein have also been used for the preservation of fruits and vegetables (Marcos et al., 2007; Del Nobile et al., 2009). Thus, zein is a promising material in biomedical applications (Liu et al., 2005; Bai, 2007; Yao et al., 2007). No research has been reported, however, concerning the application of zein scaffold in periodontal tissue engineering. In this study, we investigated the use of zein as a suitable scaffold material for periodontal tissue regeneration.

\section{Materials and Methods}

\section{Reagents and instruments}

Zein (Wuhan Hezhong Biochemical Manufacturing Co., Ltd., China), sodium chloride $(\mathrm{NaCl})$ analytical reagent, and anhydrous ethanol analytical reagent were from commercial sources. Dulbecco's modified Eagle Medium (DMEM) and trypsin were from Gibco (Invitrogen, USA). Fetal bovine serum was from Sijiqing (Hangzhou, China). The scanning electron microscope was from HITACHI (S-3500N, Japan), and the inverted phase contrast microscope was an Olympus IMT-2 (Olympus, Japan). Rhizoma coptidis, Radix scutellariae, and Rhizoma drynariae were provided by the Dispensary of Traditional Chinese Medicine in the Fourth Hospital of the Hebei Medical University.

\section{Preparation of zein scaffold}

Zein was dissolved in $65 \%$ alcohol. $\mathrm{NaCl}$ was added to the zein solution, and the resulting mixture was heated with stirring until $\mathrm{NaCl}$ was evenly dispersed into the gel system. The zein gel was cast into a certain size in the vessel by incubating at $85^{\circ} \mathrm{C}$ for $14-18 \mathrm{~h}$. Subsequently, the zein gel was placed in deionized water at $37^{\circ} \mathrm{C}$ and the water was changed every 4 hours. When no precipitation was detected via silver nitrate, leaching was allowed to continue for another $24 \mathrm{~h}$. Finally, the gel was dried at $37^{\circ} \mathrm{C}$ in a biochemical incubator, and porous zein scaffold was obtained. Under the same size of $\mathrm{NaCl}$ particle $(180-250 \mu \mathrm{m})$, three different mass concentrations of $\mathrm{NaCl}(70 \%$, $75 \%$, and $80 \%$ ) were prepared for zein scaffolds to measure the relationship between the concentration of $\mathrm{NaCl}$ and scaffold porosity, as described in the pycnometer method (Shi et al., 2001). Meanwhile, under $80 \%$ mass concentration of $\mathrm{NaCl}$, three different sizes of $\mathrm{NaCl}$ particles $(180-250 \mu \mathrm{m}$, 250-300 $\mu \mathrm{m}, 300-425 \mu \mathrm{m})$ were used for the zein scaffold in order to observe the relationship between $\mathrm{NaCl}$ particles and scaffold pore size with the scanning electron microscopy.

\section{Preparation of scaffold extract and liquid extraction experiment}

Based on the equivalent of $3 \mathrm{~cm}^{2}$ total surface area per $1 \mathrm{ml}$ extracting medium, as per the criteria of GB/T16886.12-2005, scaffolds were transferred into DMEM medium with $10 \% \mathrm{FBS}$, to be placed into a $37^{\circ} \mathrm{C} / 5 \% \mathrm{CO}_{2}$ incubator for $72 \mathrm{~h}$, obtaining $100 \%$ scaffold extract. The $100 \%$ scaffold extract was followed by serial dilutions using DMEM with $10 \%$ FBS to a final concentration of $10 \%$, $50 \%$, and $100 \%$.

An ambush mandibular third molar was selected to scrape the middle $1 / 3$ of periodontal ligament root. The samples were grown in primary culture as described by the tissue method (Situ and $\mathrm{Wu}$, 1996). The third passage cells were seeded in 96-well plates at $1 \times 10^{3} / 100 \mu \mathrm{L}$ per well, and grown in the $37^{\circ} \mathrm{C} / 5 \% \mathrm{CO}_{2}$ incubator for $24 \mathrm{~h}$. Culture medium was removed and cells were randomly divided into three groups: experimental group (containing $10 \%, 50 \%$, and $100 \%$ of scaffold extract), positive control group (containing DMEM with $0.64 \%$ phenol), and negative control group (containing DMEM alone). Thirty wells were randomized to the groups, with six wells each in the positive and negative control groups, respectively. The other 18 wells were randomly distributed to 
$10 \%, 50 \%$, and $100 \%$ of scaffold extract, with six wells per concentration. All samples were cultured in the $37^{\circ} \mathrm{C} / 5 \% \mathrm{CO}_{2}$ incubator for $24 \mathrm{~h}, 48 \mathrm{~h}$, and $72 \mathrm{~h}$. Cell viability was measured by MTT colorimetric assay, and relative growth rate (Relative Growth Rate, RGR) was calculated according to the formula of $\mathrm{RGR}=$ (absorbance A value of experimental group / absorbance A value of negative control group) $\times 100 \%$, while the toxicity of the material was evaluated based on GB/T16175-2008, as shown in Table 1.

Table 1 Cell toxicity grade

\begin{tabular}{lccccc}
\hline grade & 0 & 1 & 2 & 3 & 4 \\
\hline $\mathrm{RGR} / \%$ & $\geq 100$ & $80-99$ & $50-79$ & $30-49$ & $0-29$ \\
\hline
\end{tabular}

\section{Direct contact assay}

The zein scaffold was treated with ultraviolet (UV) for $1 \mathrm{~h}$, and soaked in DMEM solution containing $20 \%$ FBS overnight. The fourth passage cells at the concentration of $3.5 \times 10^{4} \mathrm{~mL}^{-1}$ were inoculated into the scaffold. After eighteen days, a light microscope and scanning electron microscope were used to observe the cell morphology.

\section{Shuanghuangbu preparation and Cell proli- feration assay with shuanghuangbu-scaffold extracts}

A mixture of Rhizoma coptidis, Radix scutellariae, and Rhizoma drynariae (in the proportion of $2: 1: 1$ ) was soaked in water $(8$ times and 6 times, respectively), boiled twice and filtered, and the concentration of $1 \mathrm{~g} \cdot \mathrm{mL}^{-1}$ Shuanghuangbu decoction was made. Then the Shuanghuangbu decoction was filtered and diluted with $100 \%$ scaffold extract to final concentrations $(10,25,50,100,150$, 200, 500, and $1000 \mu \mathrm{g} \cdot \mathrm{mL}^{-1}$ ), respectively. The $\mathrm{pH}$ of the final shuanghuangbu-scaffold extracts was adjusted to $7.0-7.1$, then they were stored at $4^{\circ} \mathrm{C}$.

Cells were collected and inoculated as described above, and then were randomly divided into DMEM group, scaffold extract group, and Shuanghuangbu-scaffold extracts group with different concentrations $(10,25,50,100,150,200,500$, and $1000 \mu \mathrm{g} \cdot \mathrm{mL}^{-1}$ ). Sixty wells were randomized to the groups, with six wells in the DMEM group and scaffold extract groups, respectively. The other 48 wells were distributed to the Shuanghuangbuscaffold extracts group, with six wells per concentration. The cells were cultured for $24 \mathrm{~h}, 48 \mathrm{~h}$, and
$72 \mathrm{~h}$, and then measured by MTT to compare the proliferation of each group. Experiments were repeated three times.

\section{Statistical analysis}

SPSS13.0 statistical software was used to conduct single-factor ANOVA analysis. $P<0.05$ was considered as the threshold for a statistically significant difference.

\section{Results}

\section{Physical properties of zein scaffolds}

The zein scaffold displayed a high porosity with a sponge-like structure, and the pores on the zein scaffold were distributed evenly. Their shape was variable, but mostly round and interconnected. Furthermore, the pore sizes were mostly similar, but scattered with relatively large ones (Figure 1).

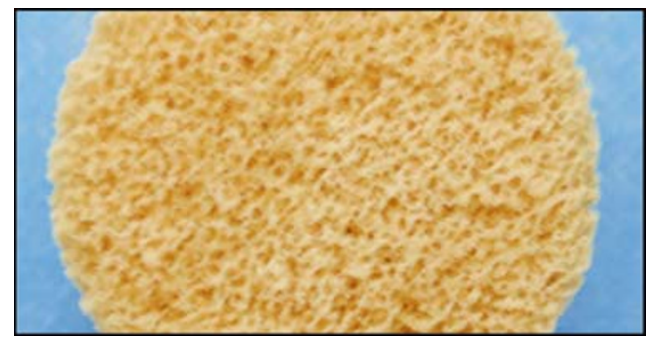

Figure 1 The photo of zein scaffold

Zein scaffold has a sponge-like structure, with pores welldistributed.

With the same size of the porogens, zein scaffold porosity was closely related to the percentage of porogen. As the percentage of porogen increased, the porosity of scaffolds increased accordingly (Table 2).

Table 2 Relationship between the porosity of scaffolds and the percentage of porogen (mean $\pm \mathrm{SD}$ )

\begin{tabular}{ccc}
\hline Percentage/ \% & $n$ & Porosity/ \% \\
\hline 70 & 30 & $64.1 \pm 2.7$ \\
75 & 30 & $70.5 \pm 2.6$ \\
80 & 30 & $78.0 \pm 2.2$ \\
\hline
\end{tabular}

Under the same amount of porogen, zein scaffolds showed the porous network structure. The sizes between pores and particles of porogen were clearly correlated. As the porogen particle diameter increased, the size of the pores also increased (Figure 2). In addition, a few microns 

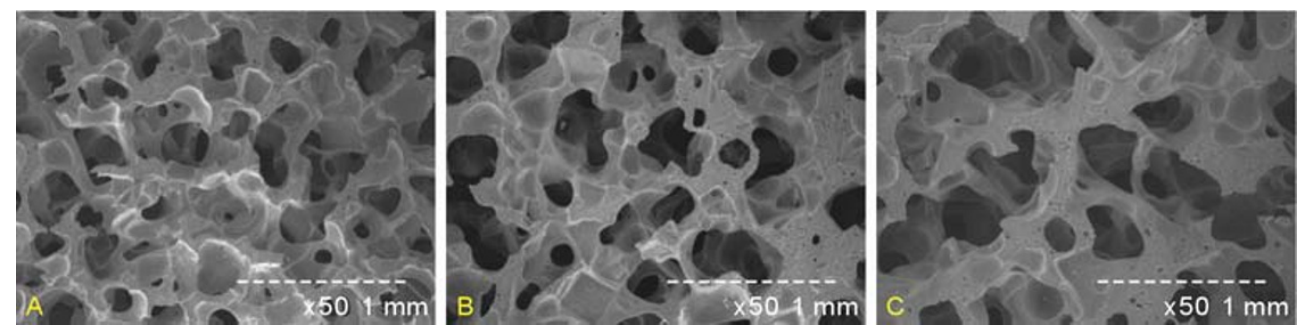

Figure 2 The size of porogen were (A) 180-250 $\mu \mathrm{m}$, (B) 250-300 $\mu \mathrm{m}$, (C) 300-425 $\mu \mathrm{m}(\mathrm{SEM}, \times 50)$ The size of scaffold pores increased when porogen particle diameter increased.

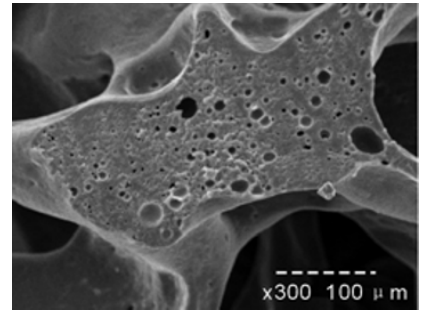

Figure 3 Microns pores observation $(\mathrm{SEM}, \times 300)$

Microns pores were scattered in scaffold walls.

to tens of microns-sized pores were distributed in scaffold walls, being beneficial by acting as conduits among pores, and increasing the roughness of pore walls (Figure 3).

\section{Liquid extraction experiment}

Zein scaffolds were biologically evaluated by cytotoxicity tests which were carried out based on liquid extraction experiment and direct contact assays. The results of liquid extraction experiment showed that for culturing $24 \mathrm{~h}, 48 \mathrm{~h}$, and $72 \mathrm{~h}$, the positive control and the negative control had a significant difference $(P<0.01)$. The experimental group and negative control group showed no significant difference $(P>0.05)$. Based on GB/T161752008 , the cell toxicity of phenol was rated as $3-4$ grades, but the cell toxicity of zein scaffolds was rated as the $0-1$ grade (Table 3 ).

\section{Direct contact assay}

These cells displayed the elongated spindle-like shape and were grown along the pores under observation with the inverted phase contrast microscope. More periodontal ligament cells showed a tight attachment to zein scaffolds under scanning electron microscope. These cells were fully stretched, starshaped, or spindle-shaped, extending several pseudopodia-like protrusions along the edge of the pore, and closely linked with the surrounding cells (Figure 4).

Table 3 Toxicity grade for zein scaffold (mean \pm SD)

\begin{tabular}{|c|c|c|c|c|c|c|c|c|c|}
\hline \multirow{2}{*}{ Group } & \multicolumn{3}{|c|}{$24 \mathrm{~h}$} & \multicolumn{3}{|c|}{$48 \mathrm{~h}$} & \multicolumn{3}{|c|}{$72 \mathrm{~h}$} \\
\hline & A value & RGR & Grade & A value & RGR & Grade & A value & RGR & Grade \\
\hline DMEM (negative) & $0.441 \pm 0.030$ & 100 & 0 & $0.464 \pm 0.021$ & 100 & 0 & $0.537 \pm 0.017$ & 100 & 0 \\
\hline $10 \%$ extract & $0.440 \pm 0.023$ & 100 & 1 & $0.467 \pm 0.018$ & 105 & 0 & $0.541 \pm 0.017$ & 100 & 0 \\
\hline $50 \%$ extract & $0.446 \pm 0.012$ & 107 & 0 & $0.475 \pm 0.012$ & 111 & 0 & $0.540 \pm 0.020$ & 106 & 0 \\
\hline $100 \%$ extract & $0.443 \pm 0.018$ & 100 & 0 & $0.472 \pm 0.012$ & 111 & 0 & $0.543 \pm 0.019$ & 108 & 0 \\
\hline Phenol (positive) & $0.199 \pm 0.013^{a}$ & 45 & 3 & $0.177 \pm 0.012^{a}$ & 38 & 3 & $0.123 \pm 0.012^{\mathrm{a}}$ & 23 & 4 \\
\hline
\end{tabular}

extracts $v s$ negative control, $P>0.05$; positive control $v s$ negative control, ${ }^{\mathrm{a}} P<0.01$.
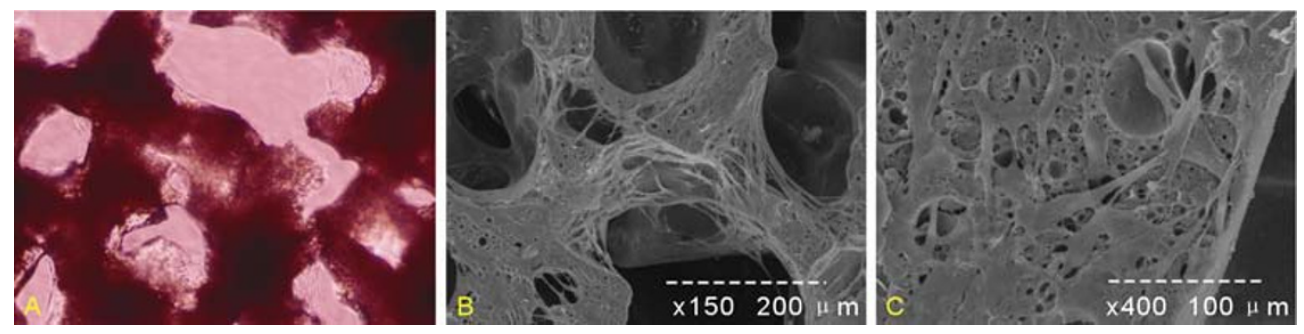

Figure 4 Periodontal ligament cells attached to zein scaffolds

A: inverted phase contrast microscope, $\times 100$; B: SEM $\times 150$; C: SEM, $\times 400$. PDLCs grew along the pores and tight attached to zein scaffolds. 
Table 4 The effects of shuanghuangbu-scaffold extracts at various concentrations on the proliferation of periodontal ligament cells (mean $\pm \mathrm{SD})$

\begin{tabular}{lcccc}
\hline \multicolumn{1}{c}{ Group } & $\begin{array}{c}\text { Shuanghuangbu } \\
\text { concentrations } / \mu \mathrm{g} \cdot \mathrm{mL}^{-1}\end{array}$ & $24 \mathrm{~h}$ & $48 \mathrm{~h}$ & $72 \mathrm{~h}$ \\
\hline DMEM & 0 & $0.336 \pm 0.027$ & $0.429 \pm 0.029$ & $0.453 \pm 0.020$ \\
scaffold extract & 0 & $0.336 \pm 0.012$ & $0.430 \pm 0.012$ & $0.456 \pm 0.019$ \\
shuanghuangbu-scaffold & 10 & $0.356 \pm 0.031$ & $0.441 \pm 0.025$ & $0.467 \pm 0.021$ \\
extracts & 25 & $0.362 \pm 0.032^{\mathrm{bc}}$ & $0.436 \pm 0.042$ & $0.473 \pm 0.032$ \\
& 50 & $0.360 \pm 0.026^{\mathrm{ac}}$ & $0.453 \pm 0.024^{\mathrm{ac}}$ & $0.477 \pm 0.021^{\mathrm{a}}$ \\
& 100 & $0.373 \pm 0.029^{\mathrm{bd}}$ & $0.458 \pm 0.016^{\mathrm{bd}}$ & $0.483 \pm 0.019^{\mathrm{bd}}$ \\
& 150 & $0.338 \pm 0.013$ & $0.444 \pm 0.015$ & $0.478 \pm 0.018^{\mathrm{ac}}$ \\
& 200 & $0.344 \pm 0.023$ & $0.443 \pm 0.025$ & $0.463 \pm 0.018$ \\
& 500 & $0.338 \pm 0.014$ & $0.436 \pm 0.026$ & $0.455 \pm 0.019$ \\
& 1000 & $0.337 \pm 0.011$ & $0.432 \pm 0.015$ & $0.454 \pm 0.036$ \\
\hline
\end{tabular}

Scaffold extract vs DMEM, $P>0.05$; shuanghuangbu-scaffold extracts $v$ s scaffold extract, ${ }^{\mathrm{a}} P<0.05,{ }^{\mathrm{b}} P<0.01$; shuanghuangbu-scaffold extracts $v$ DMEM, ${ }^{\mathrm{c}} P<0.05,{ }^{\mathrm{d}} P<0.01$.

\section{Cell proliferation assay with Shuanghuangbu- scaffold extracts}

Results showed that, compared with DMEM group, the scaffold extract group had no significant difference in terms of PDLC growth $(P>0.05)$. Compared with the DMEM group and the scaffold extract group, different concentrations of the Shuanghuangbu-scaffold extract could enhance cell proliferation differently, but $100 \mu \mathrm{g} \cdot \mathrm{mL}^{-1}$ had statistically the most significant effect $(P<0.01)$, Table 4 .

\section{Discussion}

Periodontal tissue engineering techniques for generating scaffold material are an important topic in the field of dental research. In this paper, zein, a corn protein, was used as a scaffold material, and its suitability for tissue engineering was explored. We demonstrated that zein scaffold may be feasible for periodontal tissue engineering through liquid extraction experiments, the direct contact assay, and cell proliferation assay with shuanghuangbu-scaffold extracts. Also, these results led us to conclude that zein scaffold has a definite potential for biomedical applications.

Zein protein has been studied in many areas. For example, Dong (Dong et al., 2004) successfully applied zein to human liver cells (HL-7702) and rodent fibroblasts (NH3T3). Heparin-loaded zein microsphere film and its degraded product had better biocompatibility when they were used to culture human umbilical vein endothelial cells (Wang et al., 2005). Gong (Gong et al., 2006) further demonstrated that zein scaffold can promote rat bone marrow stromal cell adhesion, growth, and differentiation, suggesting that zein protein possesses good histocompatibility (Wang et al., 2007). Here zein as a scaffold material was studied for periodontal tissue engineering. Results showed that the porosity of the zein scaffold was $64.1 \%$ $78.0 \%$, and increased as the percentage of the $\mathrm{NaCl}$ porogen increased. The scaffold diameter increased when the porogen particle size increased. Thus, one can prepare zein scaffolds with different porosity and pore size by adjusting the percentage and particle size of porogens. In addition, results from SEM showed that many micropores with the diameter of a few microns to a few tens of microns were distributed on the scaffold walls, improving the linkage between the porosities, and also enhancing the roughness of the scaffold surface, which is conducive to cell growth and the integration of cell organization (Anselme, 2000).

In order to verify if zein scaffold benefits cell growth, a toxic assay on cell growth was conducted. Periodontal ligament cells grown in primary culture was used in this study and two kinds of experiments including the liquid extraction experiment and the direct contact assay were carried out. Results showed that these scaffold extracts had no effect on the activity of PDLC proliferation and no toxic reaction either. PDLCs stretched out completely, displayed multiple pseudopodia-like protrusions trespassing the mcirohole surface, or along the 
microhole wall on the scaffold materials, as observed under SEM in the direct contact assay. This indicates that the scaffold materials had good cell compatibility and no potential toxicity.

There are a number of ways to prepare the scaffolds (Gomes et al., 2006; Petrie Aronin et al. 2008; Wei et al., 2009). The solution casting/ particulate leaching method is the most popular one, because of the simple equipment, controllable pore size, and porosity. However, there are several shortcomings in this method, such as the less than $2 \mathrm{~mm}$ thickness of the scaffold caused by the particle deposition, the lower porous nature of inter-linking, and the organic solvent residues left that may affect cell growth. In order to overcome these shortcomings, some researchers have suggested approaches (Murphy et al., 2002). We has modified some procedures by using the heating system during stirring, which creates a uniform distribution of porogen gelatinous, casting small settlement after the porogens, and creating more than a $2 \mathrm{~mm}$ frame thickness of the scaffold. In addition, waterbath heating was used to promote evaporation of alcohol in a relatively short period of time. Volatile elements during the overflow went through the channel and increased the inter-linking nature of the pore. Alcohol has been used because of its low toxicity. When used with heat, alcohol was evaporated and cell toxicity caused by the residual amount was low.

Periodontal tissue repair and reconstruction is closely related to periodontal ligament cell proliferation and differentiation. Whether periodontal tissue engineering can proceed smoothly or not lies in the activity of PDLC proliferation and differentiation. Some studies have shown that a variety of growth factors are involved in strengthening cell chemotaxis function (Zaman et al., 1999), but the effect of applying a single growth factor is not always good (Oates et al., 1993). Thus, several growth factors must be combined in spite of those high prices. In recent years, studies have shown that the Chinese medicine Shuanghuangbu can prompt the proliferation, differentiation, and protein synthesis of PDLCs (Xu et al., 2004; Xu et al., 2009). Shuanghuangbu can obviously promote new bone and cementum formation, restrain the migration of junctional epithelium, and enhance the regeneration of periodontal tissue $(\mathrm{Xu}$ et al., 2005); Shuanghuangbu sustained-release preparation can significantly improve the clinical symptom of periodontitis and change the composition of subgingival microflora at disease sites to that at healthy sites (Xu et al., 2004). Thus, we thought that applying Shuanghuangbu as a growth factor to periodontal tissue engineering may achieve a satisfactory effect. This research found that different concentrations of the Shunghuangbu-scaffold extract could enhance cell proliferation to various degrees, but $100 \mu \mathrm{g} \cdot \mathrm{mL}^{-1}$ showed statistically the most significant effect. These findings provide an objective basis for consideration of zein-shuanghuangbu-PDLC complex for periodontal tissue regeneration in clinical applications.

\section{Conclusions}

We conclude that zein scaffold has high porosity, opened pore wall structure, and non-toxicity. The good biocompatibility is conducive to the growth of PDLCs. These results provide the evidence of zein's suitability as scaffold material for periodontal tissue engineering. Also, Shuanghuangbuscaffold extract can enhance the proliferation activity of PDLCs. Future studies of in vivo testing on animals are required to further clarify the combination of Shuanghuangbu-zein scaffold-cell and how to repair periodontal defects.

\section{Acknowledgrments}

This study was supported by a grant (30873289) from the Chinese National Science Foundation.

\section{References}

Anselme K (2000). Osteoblast adhesion on biomaterials. Biomaterials, 21(7): 667-681.

Bai Y (2007). The current and future applications of paper in medicine and biotechnology. World Pulp Paper, 26(6): 5-8.

Chen FM, Yan J (2010). Periodontal tissue engineering and regeneration: current approaches and expanding opportunities. Tissue Engineering: Part B, 16(2): 219-255.

Dawson JI, Wahl DA, Lanham SA, Kanczler JM, Czernuszka JT, Oreffo ROC (2008). Development of specific collagen scaffolds to support the osteogenic and chondrogenic differentiation of human bone marrow stromal cells. Biomaterials, 29(21): 3105-3116.

Del Nobile MA, Muratore G, Conte A, Incoronato AL, 
Panza O (2009). An integrated approach to extend the shelf life of a composite pastry product (Cannoli). $J$ Food Prot, 72(12): 2553-2560.

Dong J, Sun QS, Wang JY (2004). Basic study of corn protein, zein, as a biomaterial in tissue engineering, surface morphology and biocompatibility. Biomaterials, 25(19): 4691-4697.

Gomes M, Holtorf H, Reis R, Mikos A (2006). Influence of the porosity of starch-based fiber mesh scaffolds on the proliferation and osteogenic differentiation of bone marrow stromal cells cultured in a flow perfusion bioreactor. Tissue Eng, 12(4): 801-809.

Gong SJ, Wang HJ, Sun QS, Xue ST, Wang JY (2006). Mechanical properties and in vitro biocompatibility of porous zein scaffolds. Biomaterials, 27(20): 3793-3799.

$\mathrm{Ku} \mathrm{K}$, Song KB (2007). Physical properties of nisinincorporated gelatin and corn zein films and antimicrobial activity against Listeria monocytogenes. $J$ Microbiol Biotechnol, 17(3): 520-523.

Liu XM, Sun QS, Wang HJ, Zhang L, Wang JY (2005). Microspheres of corn protein, zein, for an ivermectin drug delivery system. Biomaterials, 26(1): 109-115.

Marcos B, Aymerich T, Monfort JM (2007). Use of antimicrobial biodegradable packaging to control Listeria monocytogenes during storage of cooked ham. Int $J$ Food Microbiol, 120 (1/2): 152-158.

Montjovent MO, Mark S, Mathieu L, Scaletta C, Scherberich A, Delabarde C, et al. (2008). Human fetal bone cells associated with ceramic reinforced PLA scaffolds for tissue engineering. Bone, 42(3): 554-564.

Murphy W, Dennis R, Kileny J, Mooney D (2002). Salt fusion: an approach to improve pore interconnectivity within tissue engineering scaffolds. Tissue Eng, 8(1): $43-52$.

Oates TW, Rouse CA, Cochran DL (1993). Mitogenic effects of growth factors on human periodontal ligament cells in vitro. J periodontal, 64 (2): 142-148.

Petrie Aronin CE, Cooper JA Jr, Sefcik LS, Tholpady SS, Ogle RC, Botchwey EA (2008). Osteogenic differentiation of dura mater stem cells cultured in vitro on threedimensional porous scaffolds of poly(e-caprolactone) fabricated via co-extrusion and gas foaming. Acta Biomater, 4(5): 1187-1197.

Shi GX, Wang SG, Bei JZ (2001). Preparation and spatial superficial syndrome of the porous cell cytoskeletal with polylactic acid and polylactic acid-hydroxyacetic acid. J Funcl Polymers, 14(3): 7-11.

Situ ZQ, Wu JZ (1996). Cell culture. Xi'an: World Book Publishing House, pp193-194.

Thein-Han WW, Misra RD (2009). Biomimetic chitosannanohydroxyapatite composite scaffolds for bone tissue engineering. Acta Biomater, 5(4): 1182-97.

Wang HJ, Gong SJ, Lin ZX, Fu JX, Xue ST, Huang JC, et al. (2007). In vivo biocompatibility and mechanical properties of porous zein scaffolds. Biomaterials, 28(27): 3952-3964.

Wang HJ, Lin ZX, Liu XM, Sheng SY, Wang JY (2005). Heparin-loaded zein microsphere film and hemocompatibility. J Control Release, 105(1/2): 120-131.

Wei J, Chen FP, Shin JW, Hong H, Dai CL, Su JC et al. (2009). Preparation and characterization of bioactive mesoporous wollastonite - polycaprolactone composite scaffold. Biomaterials, 30(6): 1080-1088.

Xu YZ, Yang GY, Luo DQ (2009). Effects of Shuanghuangbu on proliferation activity of human periodontal ligament cells cultured. J Clin Reh Tissue Eng Res, 13(37): 7341-7345.

Xu YZ, Zou HR, Wang XL, Liu SZ Wang YJ (2004). Effects of Shuanghuangbu on the total protein content and ultrastructure in cultured human periodontal ligament cells. Chin Med J (Eng1), 117(11): 16931696.

Xu YZ, Liu J, Li W, Wang XL, Liu SZ (2004). Clinical study of Shuanghuangbu delivery device in chronic periodontitis. J Modern Stomatol, 18(1): 46-49.

Xu YZ, Zhang DM, Wang XL, Wang YJ (2005). The study of Shuanghuangbu delivery device in experimental regeneration of periodontium. $J$ Chongqing Medical University, 30(1): 78-81.

Yao C, Li XS, Song TY (2007). Fabrication of zein/ hyaluronic acid fibrous membranes by electrospinning. J Biomate Sci Polym Ed, 18(6): 731-742.

You ZY, Cushman M, Jenny NS, Howard G (2009). Tooth loss, systemic inflammation, and prevalent stroke among participants in the reasons for geographic and racial difference in stroke (REGARDS) study. Atherosclerosis, 203(2): 615-619.

Zaman KU, Sugaya T, Kato H (1999). Effect of recombinant human platelet-derived growth factor-BB and bone morphogenetic protein-2 application to demineralized dentin on early periodontal ligament cell response. J Periodontal Res, 34(5): 244-250.

\footnotetext{
*Corresponding author: Yan-zhi Xu

Address: Department of Stomatology, the Fourth Hospital of Hebei Medical University, Shijiazhuang 050011, China

Tel: 86031186095436 E-mail:xu_yanzhi@163.com
} 